\title{
Robust Controller Design for the Cooperative Control of Lower Extremity Rehabilitation Robot
}

\author{
Zhizheng Wu, Feng Li, Wenjun Mi and Jingtao Lei \\ Department of Precision Mechanical Engineering, Shanghai University \\ zhizhengwu@shu.edu.cn
}

\begin{abstract}
In this paper, a robust controller design approach is proposed for Lower Extremity Rehabilitation Robot (LERR) with automatic gait adaption. Based on the developed LERR dynamic model, the robot system is first decoupled into some independent second-order integral systems using inverbility decoupling method. Then a robust optimal PID controller is proposed for each sub-system to cope with the uncertainty in the system modeling by converting the PID controller design problem into a static output feedback $\mathrm{H}_{2} / \mathrm{H}_{\infty}$ multiobjective optimization problem. The adaptive control methods are further investigated for a compliant gait trajectory planning. The performance of the designed controller is finally verified in the Adams-MATLAB/Simulink environment to show the effectiveness of the proposed robust control approach.
\end{abstract}

Keywords: rehabilitation robot; robust; cooperative; adaptive control

\section{Introduction}

It has been proved that task-oriented repetitive movements can improve muscular strength and coordination for patients with hemiplegia or stroke and other limb movement disorders due to brain plasticity $[1,2]$. Treadmill training has been used for more than ten years as a regular therapy. However manual-assisted treadmill training has several limitations such as labor-intensive, low security and low repeatability, etc. In contrast, these shortcomings mentioned above could be overcome with automated gait training, for example, using the robotic gait orthosis [3, 4]. Normally, the robotic gait trainers work in two ways with respect to the cooperation with the patients. In the "no-cooperative" way the robot does not adapt the movement of the joints to the activity of the patient, whose limbs will be moved along a predefined fixed trajectory. In the "patient-cooperative" way the robot control system will recognize the patient's movement intention, then feedback the information and adapt the robotic assistance to the patient's contribution. For the robotic gait orthosis Locomat [4], the impedance control and the admittance control methods have been considered in the control system to cooperate the patient's intentions. In [5] the control system of the Robotic Gait Rehabilitation Trainer with a novel mechanical design is presented, which targets secondary gait deviations affecting patterns of movement of the pelvis in stroke survivors. Similarly, an expanded impedance control strategy is applied to generate a force field that affects the obliquity of the pelvis via a lower body exoskeleton while the patient ambulates on a treadmill. In [6] a bidirectional human-machine interface including a neurofuzzy controller and an extended physiological proprioception feedback system is developed by imitating the biological closed-loop control system of 
human body. The interface rebuilds a closed-loop motion control system for paralyzed patients and realizes harmonious control of the human-machine system. In [7] a Virtual Model Controller (VMC) with an impedance control method is used to offer gait assistance. The developed VMC provides virtual support at the ankle to increase foot clearance and the impedance levels were then altered between trials to investigate whether the controller allowed gradual and selective support. A recent review of the current state of art control approaches for the rehabilitation robots can be found in [8].

The patient cooperative control architecture for the rehabilitation robot normally consists of two control loops - inner and out loops. The inner loop is implemented to control each joint to follow the desired trajectory individually, in which the PD or PID controllers with inverse robot dynamic compensation are used. The out loop controller is then designed to modify the predefined joint trajectories based on the information extracted from the patient-robot interactive interface. The control design method for the out control loop has been rigorously studied in the literature, while the design method for the inner control loop is usually omitted since it is a well-known traditional robot control area. However, for the lower extremity rehabilitation robot system, the system dynamic model normally cannot be developed accurately due to the different physical parameters in the patients. Therefore, it is highly desirable to consider the robustness for the inner control loop design. A six-degrees-of-freedom robotic orthosis called Lower Extremity Rehabilitation Robot (LERR) (Figure 1) has been built in Precision Mechanical Lab at Shanghai University. The dynamic model of LERR was developed and verified in [9]. In this paper, the automation gait pattern adaption problem for LERR with patient interactions is studied based on a robust controller design approach. The purpose of this study is to recognize the patient's movement intention and then realize active control under the awareness of the initiative with the assumption that the dynamic model of the robot system is inaccurate. The robot system is first decoupled using invertibility decoupling theory, then based on the decoupled two order integral sub-systems, a decentralized robust PID controller is developed to optimize the position tracking performance and cope with the uncertainty in the patient-robot cooperative system. In order to make LERR walk more patient cooperative, an adaptive control algorithm is further developed to adapt the reference gait pattern by reducing the interaction torques between the robot and patient and, thus, allow the patient to influence the gait pattern cooperatively. The performance of the designed controller is verified in the Adams-MATLAB/Simulink environment to show the effectiveness of the proposed robust control approach.

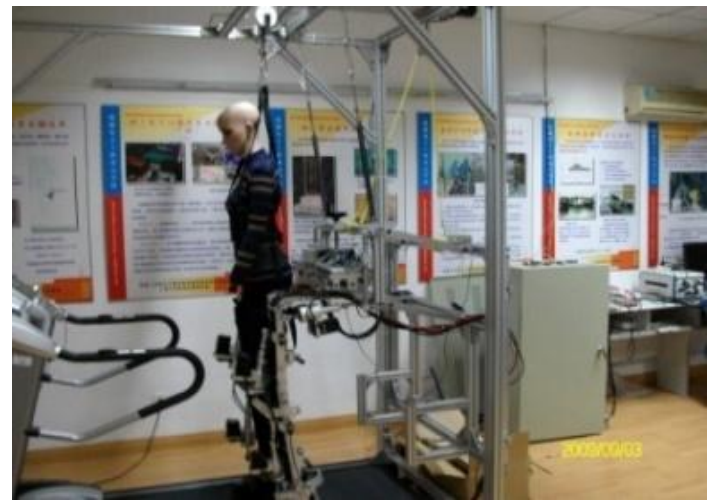

Figure 1. Lower Extremity Rehabilitation Robot 


\section{Controller Design}

In this section, the robust controller design approach is presented. Firstly, the robot system is decoupled into three decoupled two order integral sub-systems using invertibility decoupling theory. Then based on the decoupled subsystems, a decentralized robust PID controller is developed in the inner control loop to optimize the trajectory tracking performance and cope with the uncertainty in the patient-robot cooperative system. In order to deal with the patient cooperative intention, an adaptive control algorithm is further developed in the out control loop to adapt the reference gait pattern and allow the patient to influence the gait pattern cooperatively.

\subsection{Invertibility decoupling}

LERR system is a complicated switched nonlinear system. Since the gait cycle is divided into stance phase and swing phase, the dynamics model can be written as:

$$
\Sigma_{r}\left\{\begin{array}{l}
M_{r}(\theta) C_{r}(\theta, \theta) G_{r}(\theta)=\tau \\
r=1, \quad \text { in swing phase } \\
r=2, \quad \text { in stance phase. }
\end{array}\right.
$$

where $M_{r}$ is a $3 \times 3$ inertia matrix, $C_{r}$ is a $3 \times 3$ centrifugal force and Coriolis force matrix, $G_{r}$ is a $3 \times 1$ gravity matrix, and $\tau$ is $3 \times 1$ joint torque required by mechanical leg. $\tau=\tau_{m}+\tau_{a c t}, \tau_{m}$ is the motor drive torque and $\tau_{a c t}$ is the patient active torque. In swing phase, $r=1$, and in stance phase, $r=2$.

Using the standard invertibility decoupling method [10,11], the system could be decoupled into three independent second-order integral systems. For the dynamics model in (1) , we define:

$$
R_{r 0}=M_{r}(\theta)^{-1}, R_{r 1}=G_{r}(\theta), R_{r 2}=C_{r}(\theta, \theta)
$$

If we denote $X_{1}=\theta, X_{2}=\theta$ and $Y=X_{1}$, the system then transforms into the following state space form as:

$$
\left\{\begin{array}{l}
{\left[\begin{array}{l}
X_{1}^{\&} \\
X_{2}^{\&}
\end{array}\right]=\left[\begin{array}{c}
X_{2} \\
-R_{r 0} *\left(R_{r 2} X_{2}+R_{r 1}\right)
\end{array}\right]+\left[\begin{array}{c}
0 \\
R_{r 0} * \tau
\end{array}\right]} \\
\left\{\begin{array}{cc}
r=1, & \text { in swing phase } \\
r=2, & \text { in stance phase. }
\end{array}\right.
\end{array}\right.
$$

Take the derivative of $Y=X_{1}$ as

$$
Y^{\&}=X_{1}^{\&}=X_{2}
$$

Continue to take the derivative of $Y$ and we obtain

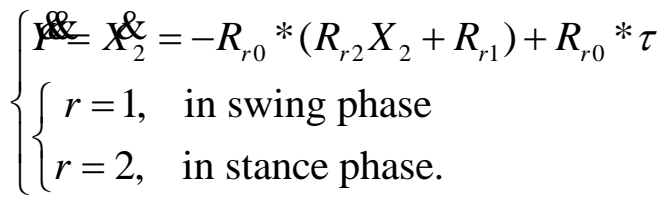

Since $R_{r 0}$ is nonsingular, the system can be decoupled into 3 independent second-order integral systems as: 


$$
\mathrm{v}_{i}(\mathrm{t})=(\mathrm{t}), i=1,2,3
$$

The original control system can be converted into the following form:

$$
\Sigma_{c}\left\{\begin{array}{l}
u(t)=M_{r}(\theta) V(t)+C_{r}(\theta, \theta) \&+G_{r}(\theta)-\tau_{a c t} \\
\begin{cases}r=1, & \text { in swing phase } \\
r=2, & \text { in stance phase. }\end{cases}
\end{array}\right.
$$

where $u(t)$ is the motor drive torque $\tau_{m}$. The resulting decoupled closed-loop system is then $\frac{1}{s^{2}} I_{3 \times 3}$ as shown in Figure 2 .

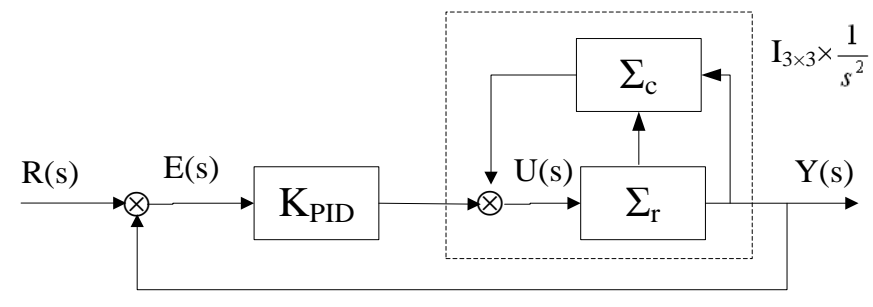

Figure 2. Block diagram of the decoupled system in the inner control loop

Though LERR is a nonlinear and strongly coupled system, based on the invertibility controller (5), the original system (1) is finally decoupled into three independent double integrator sub-systems $1 / s^{2}$ and can be denoted as $\bar{G}(s)=1 / s^{2} I$, where $I$ is a 3 by 3 indentity matrix. Then for each channel, a decentralized classical PID position controller can be easily developed to make the output $Y$ effectively track the reference trajectory $R$. However, in reality it is difficult to establish an accurate dynamic model for LERR and the decoupling will not be complete, therefore, in the following, a robust PID controller design approach is proposed, then an adaptive control is applied in the out loop to achieve a compliant patient cooperative strategy.

\subsection{Robust PID controller design}

The above decoupling approach is based on the assumption that the dynamic model of the robot system is exactly known, however, in reality it is normally difficulty to obtain the model of the whole robot system with all accurate parameters. In this case, the resulting robot system $\bar{G}(s)$ using the feedback controller (5) will not be fully decoupled. Reformulated $\bar{G}(s)$ as:

$$
\begin{aligned}
\bar{G}(s) & =\bar{g}(s) I+(\bar{G}(s)-\bar{g}(s) I) \\
& =\bar{g}(s) I+\bar{\Delta}(s),
\end{aligned}
$$

where $\bar{\Delta}(s)$ represents the additive model uncertainty and $\bar{g}(s)=1 / s^{2}$. In order to simplify the design of the PID controller, the uncertainty term $\bar{\Delta}(s)$ will be represented using an unstructured constant matrix $\Delta$ weighted by a band pass filter $w_{\Delta}(s)$ as: 


$$
\bar{\Delta}(s)=w_{\Delta}(s) \Delta
$$

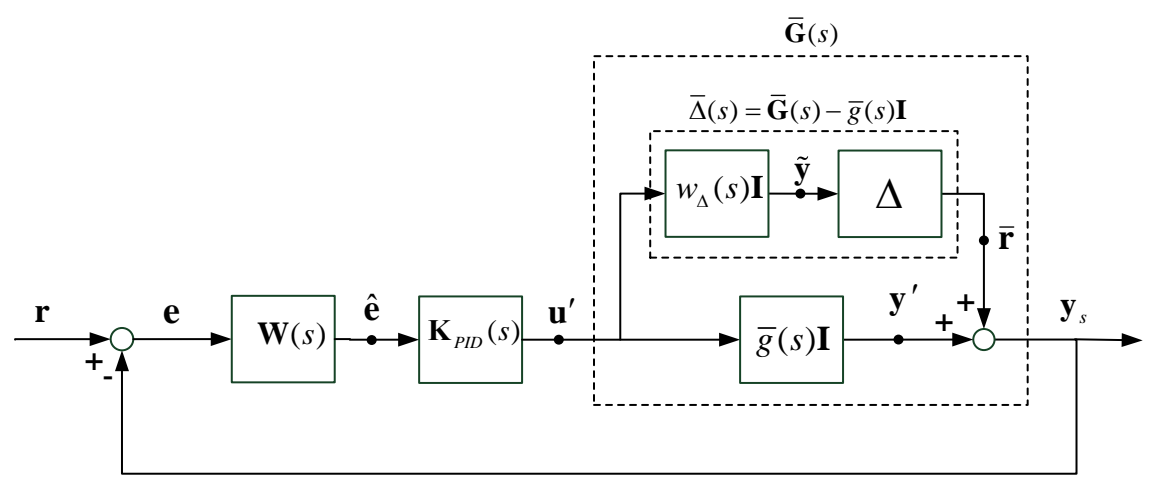

Figure 3. Block diagram of the decoupled closed-loop system with uncertain plant model

where $\Delta \in \mathrm{R}^{m \times l}$ is such that all entries $\delta^{i j}, i=1, \ldots, m, j=1, \ldots, l$ in $\Delta$ satisfy $-\rho \leq \delta_{i j} \leq \rho$ and where $\rho$ can be determined experimentally. In the following, a robust PID controller will be developed for the augmented system (6). First, in order to eliminate the high frequency noise in the real system, the filter given by $W(s)=w(s) I$, where $w(s)$ is a low pass filter transfer function, will be applied to the error signal $e$. By considering a decentralized PID controller of the form $K_{P I D}=k_{P I D} I$, it follows that the nominal closed loop system is fully decoupled. For the purpose of stability robustness analysis, the signal $r$ is set to zero first and the closed loop system block diagram is redrawn as shown in Figure 3, where $\Sigma$ is the system with input $\tilde{r}$ and output $\tilde{y}$. The system $\Sigma$ is given by

$$
\Sigma=-w_{\Delta} K_{P I D} W S_{0},
$$

where $S_{0}$ is the sensitivity function given by

$$
\begin{aligned}
S_{0} & =\left(I+\bar{g} K_{P I D} W\right)^{-1} \\
& =\left(1+\bar{g} k_{P I D} w\right)^{-1} I
\end{aligned}
$$

Let $s_{0}=\left(1+\bar{g} k_{P I D} w\right)^{-1}$. Then we have

$$
\Sigma=-w_{\Delta} k_{P I D} w s_{0} I
$$

Assuming $\Sigma$ is stable, then the robust stability of the closed loop system shown in Figure 4 is satisfied if [12]:

which is equivalent to

$$
\|\Delta\|_{\infty}\|\Sigma\|_{\infty}<1
$$

$$
\|\Sigma\|_{\infty}<\|\Delta\|_{\infty}^{-1} .
$$




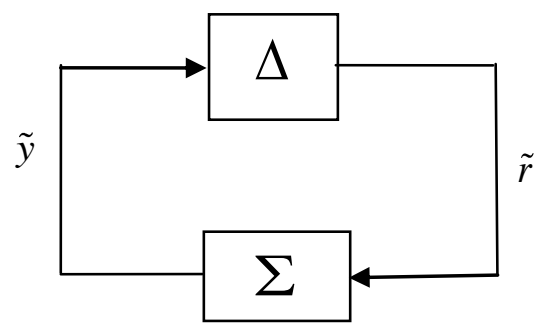

Figure 4. Block diagram of the closed loop system with $\Delta$ and $\Sigma$

Let $J \in R^{M \times L}$ be such that all its entries are equal to or less than one. Then $\|\Delta\|_{\infty} \leq \rho \sigma_{\max }(J)$, where $\sigma_{\max }(J)$ denote the maximum singular value of $J$. Based on (10), it follows that $\|\Sigma\|_{\infty}=\left\|w_{\Delta} k_{P I D} w s_{0}\right\|_{\infty}$. Let $\gamma=\frac{1}{\rho \sigma_{\max }(J)}$, then the robust stability condition of the closed loop system can be written as

$$
\left\|w_{\Delta} w k_{p i d} s_{0}\right\|_{\infty} \leq \gamma .
$$

If (12) is satisfied, then the designed PID controller yields a stable closed loop system. However, regulation performance of the closed loop system is not considered yet if the design only involves the robustness constraint (12). In the following, the convergence rate of the closed loop system with respect to the static inputs is performed by minimizing an $\mathrm{H}_{2}$ performance specification. Consider the nominal closed loop system subject to an input $r=v 1_{d}$, where $v \in \mathrm{R}^{m}$ is a constant vector and $1_{d}$ is the scalar valued unit step function. An additional controller design objective is considered where it is desired to find a PID controller $K_{P I D}$ that minimizes the $L_{2}$ norm of $e$, i.e., $\|e\|_{2}$, by considering only the nomial system $\bar{g}(s) I$ in the closed loop system (i.e., without considering the uncertainty $\bar{\Delta}(s)$ ). Since the sensitivity function $S_{0}$ relates the input $r$ to the error $e$, the solution of this design constraint can be obtained by considering a standard $H_{2}$ optimal control problem where it is desired to minimize the $\mathrm{H}_{2}$ norm of the system $\frac{1}{S} S_{0}$. In order to avoid the unstable pole introduced by $\frac{1}{s}$, the system $\frac{1}{s} S_{0}$ can be approximated by the system $\frac{1}{s+\alpha} S_{0}$, where $\alpha$ is a small positive constant. Therefore, the decentralized robust PID controller design problem is converted into a mixed $H_{2} / H_{\infty}$ multiobjective design problem as

$$
\min _{k_{\text {pid }}}\left\|\frac{1}{s+\alpha} S_{0}\right\|_{2} \text { subject to (12). }
$$

In the following, the above optimization problem will be first expressed as a mixed $\mathrm{H}_{2} / H_{\infty}$ static output feedback problem, then a synthesis algorithm based on solving 
iterative linear matrix inequalities is used to solve this nonconvex problem by obtaining a suboptimal solution. Write the following state space representation for $\bar{g}(s)$

$$
\sum_{\bar{g}}:\left\{\begin{array}{l}
\dot{x}_{g}=A_{g} x_{g}+B_{g} u_{i}^{\prime} \\
y_{i}=C_{g} x_{g}
\end{array}\right.
$$

where $x_{g}$ is the vector of state variables, $u_{i}^{\prime} \in \mathrm{R}$ is the $i^{\text {th }}$ control input, $y_{i} \in \mathrm{R}$ is the $i^{\text {th }}$ output displacement, $A_{g}, B_{g}$, and $C_{g}$ are the corresponding system matrices. A state space representation for $w(s)$ is given as follows:

$$
\sum_{w}:\left\{\begin{array}{l}
\dot{x}_{w}=A_{w} x_{w}+B_{w} e_{i} \\
\hat{e}_{i}=C_{w} x_{w}
\end{array}\right.
$$

where $x_{w}$ is the vector of state variables, $e_{i}=r_{i}-y_{i} \in \mathrm{R}$ is the $i^{\text {th }}$ performance error, $\hat{e}_{i} \in \mathrm{R}$ is the $i^{\text {th }}$ output of the filter, $A_{w}, B_{w}$, and $C_{w}$ are the corresponding system matrices. Similarly, the weight function $w_{\Delta}(s)$ and $\frac{1}{s+\alpha}$ are represented, respectively, as

$$
\sum_{\Delta}:\left\{\begin{array}{l}
\dot{x}_{\Delta}=A_{\Delta} x_{\Delta}+B_{\Delta} u_{i}^{\prime} \\
\tilde{y}_{i}=C_{\Delta} x_{\Delta}
\end{array}\right.
$$

and

$$
\sum_{s}:\left\{\begin{array}{l}
\dot{x}_{s}=-\alpha x_{s}+e_{i} \\
\tilde{e}_{i}=x_{s}
\end{array}\right.
$$

where $x_{\Delta}$ and $x_{s}$ are the vector of state variables, $A_{\Delta}, B_{\Delta}, C_{\Delta}$, and $D_{\Delta}$ are the corresponding system matrices. The control signal generated by the PID controller $k_{P I D}$ is given by

$$
u_{i}^{\prime}=k_{p} \hat{e}_{i}+k_{i} \int_{0}^{t} \hat{e}_{i} d \tau+k_{d} \dot{\hat{e}}_{i}
$$

where $k_{p}, k_{i}, k_{d} \in \mathrm{R}$ are the parameters to be designed. Let $\chi=\left[\begin{array}{lllll}\chi_{1} & \chi_{2} & \chi_{3} & \chi_{4} & \chi_{5}\end{array}\right]^{T}$, where $\chi_{1}=x_{g}, \chi_{2}=x_{w}, \chi_{3}=x_{\Delta}, \chi_{4}=x_{s}, \chi_{5}=\int_{0}^{t} \hat{e}_{i} d t$.

It follows that

$$
\begin{aligned}
& \hat{e}_{i}=\left[\begin{array}{lllll}
0 & C_{w} & 0 & 0 & 0
\end{array}\right] \chi, \\
& \int_{0}^{t} \hat{e}_{i} d t=\left[\begin{array}{lllll}
0 & 0 & 0 & 0 & 1
\end{array}\right] \chi, \\
& \dot{\hat{e}}_{i}=\left[\begin{array}{lllll}
-C_{w} B_{w} C_{g} & C_{w} A_{w} & 0 & 0 & 0
\end{array}\right] \chi+C_{w} B_{w} r_{i} .
\end{aligned}
$$


Define the new output variables $\hat{y}=\left[\hat{y}_{1}, \hat{y}_{2}, \hat{y}_{3}\right]^{T}$, where $\hat{y}_{1}=\hat{e}_{i}, \hat{y}_{2}=\int_{0}^{t} \hat{e}_{i} d \tau$, and $\hat{y}_{3}=\dot{\hat{e}}_{i}$, and the new performance variable $\left[\begin{array}{c}z_{2} \\ z_{\infty}\end{array}\right]=\left[\begin{array}{c}\tilde{e}_{i} \\ \tilde{y}_{i}\end{array}\right]$, then the state space representation from $u_{i}^{\prime}$ and $r_{i}$ to $\left[\begin{array}{c}z_{2} \\ z_{\infty}\end{array}\right]$ and $\hat{y}$ can be written as

$$
\sum_{a g}:\left\{\begin{aligned}
\dot{\chi} & =A \chi+B_{1} r_{i}+B_{2} u_{i}^{\prime} \\
{\left[\begin{array}{c}
z_{2} \\
z_{\infty}
\end{array}\right] } & =\left[\begin{array}{l}
C_{2} \\
C_{\infty}
\end{array}\right] \chi \\
\hat{y} & =C_{\hat{y}} \chi+D_{\hat{y}} r_{i}
\end{aligned}\right.
$$

where

$$
\begin{gathered}
A=\left[\begin{array}{ccccc}
A_{g} & 0 & 0 & 0 & 0 \\
-B_{w} C_{g} & A_{w} & 0 & 0 & 0 \\
0 & 0 & A_{\Delta} & 0 & 0 \\
-C_{g} & 0 & 0 & -\alpha & 0 \\
0 & C_{w} & 0 & 0 & 0
\end{array}\right], B_{1}=\left[\begin{array}{c}
0 \\
B_{w} \\
0 \\
1 \\
0
\end{array}\right], B_{2}=\left[\begin{array}{c}
B_{g} \\
0 \\
B_{\Delta} \\
0 \\
0
\end{array}\right], D_{\hat{y}}=\left[\begin{array}{c}
0 \\
0 \\
C_{w} B_{w}
\end{array}\right] \\
C_{2}=\left[\begin{array}{lllll}
0 & 0 & 0 & 1 & 0
\end{array}\right], C_{\infty}=\left[\begin{array}{lllll}
0 & 0 & C_{\Delta} & 0 & 0
\end{array}\right] \\
C_{\hat{y}}=\left[\begin{array}{ccccc}
0 & C_{w} & 0 & 0 & 0 \\
0 & 0 & 0 & 0 & 1 \\
-C_{w} B_{w} C_{g} & C_{w} A_{w} & 0 & 0 & 0
\end{array}\right] .
\end{gathered}
$$

Therefore, with respect to the system (18), the design of the PID controller is converted into a static feedback controller design problem where the control siganl is given by $u_{i}=F \hat{y}$ and where $F=\left[\begin{array}{lll}k_{p} & k_{i} & k_{d}\end{array}\right]$. The controller design problem is cast as a mixed $H_{2} / H_{\infty}$ static ouput feedback controller design problem for the system (18) based on solving the optimization problem (13). Using the static output feedback controller $F$, the state space representation of the closed loop system involving the modified plant (18) becomes

$$
\begin{aligned}
& \dot{\chi}=\left(A+B_{2} F C_{\hat{y}}\right) \chi+\left(B_{1}+B_{2} F D_{\hat{y}}\right) r_{i} \\
& \sum_{c l}:\left\{\begin{array}{l}
z_{2} \\
z_{\infty}
\end{array}\right]=\left[\begin{array}{l}
C_{2} \\
C_{\infty}
\end{array}\right] \chi
\end{aligned}
$$

It should be noted that the $H_{\infty}$ norm of the closed loop system relating $\bar{r}_{i}$ to $z_{\infty}$ (i.e. to $\tilde{y}_{i}$ ) is the same as that of the closed loop system $\Sigma_{r_{i}, z_{\infty}}$ in (19) relating $r_{i}$ to $z_{\infty}$. Also, $\left\|\frac{1}{s+\alpha} s_{0}\right\|_{2}$ is the same as the $H_{2}$ norm of the closed loop system $\Sigma_{r_{i}, z_{2}}$ in (19) relating $r_{i}$ to 
$z_{2}$ (i.e. to $\tilde{e}_{i}$ ). Consequently, the design of the PID controller presented in the following is based on considering $\left\|\sum_{r_{i}, z_{\infty}}\right\|_{\infty}$ and $\left\|\Sigma_{r_{i}, z_{2}}\right\|_{2}$. Hence, the mixed $H_{2} / H_{\infty}$ static output feedback problem can then be cast in the form of an optimization problem involving matrix inequalities as:

$$
\text { OP1: } \quad \min _{k_{p i d}} \operatorname{trace}\left(C_{2} \tilde{P} C_{2}^{T}\right)
$$

subject to

$$
\begin{gathered}
{\left[\begin{array}{cc}
\left(A+B_{2} F C_{\hat{y}}\right) \tilde{P}+\tilde{P}\left(A+B_{2} F C_{\hat{y}}\right)^{T} & \left(B_{1}+B_{2} F D_{\hat{y}}\right) \\
\left(B_{1}+B_{2} F D_{\hat{y}}\right)^{T} & -I
\end{array}\right]<0} \\
{\left[\begin{array}{ccc}
\left(A+B_{2} F C_{\hat{y}}\right) P+P\left(A+B_{2} F C_{\hat{y}}\right)^{T} & \left(B_{1}+B_{2} F D_{\hat{y}}\right) & P C_{\infty}^{T} \\
\left(B_{1}+B_{2} F D_{\hat{y}}\right)^{T} & -I & 0 \\
C_{\infty} P & 0 & -\gamma^{2} I
\end{array}\right]<0}
\end{gathered}
$$

where $\tilde{P}>0$ and $P>0$. Since the above matrix inequalities are nonlinear in the unknown parameters $\tilde{P}, P$ and $F$, they cannot be solved directly using LMI solvers. In [13] an algorithm is proposed to obtain a solution for OP1 by iteratively solving a set of properly formulated linear matrix inequalities.

\subsection{Gait adaptation}

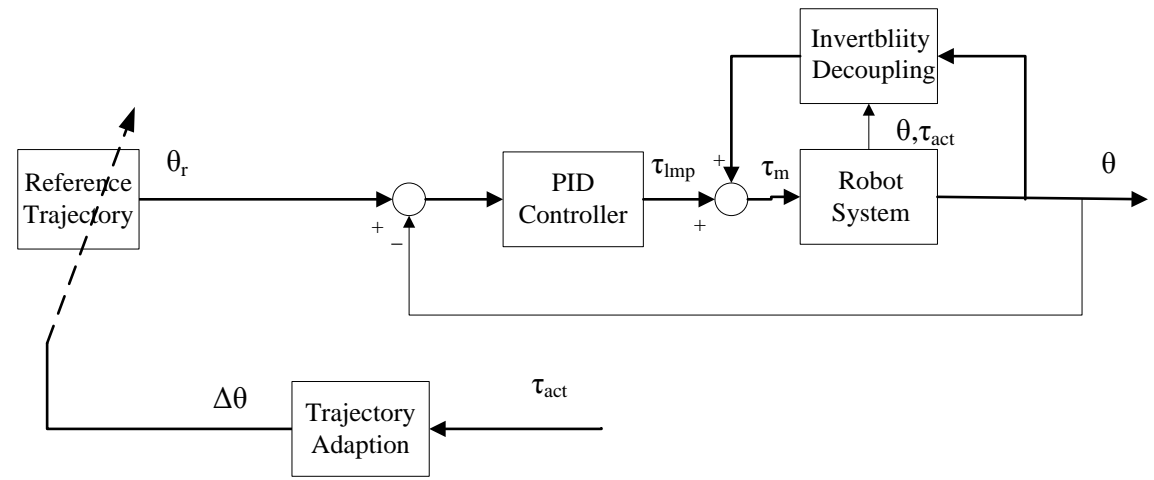

Figure 5. Trajectory adaptation control architecture

The main disadvantage of the above position control loop is that the modification of the predefined joint trajectory has to be carried out manually, and it is difficult to perform with individual adjustment. The purpose of automatic reference trajectory adaptation algorithms is to modify the robot motion optimally (in Figure 5). The new reference trajectory $\theta_{r}$ could be parameterized from nominal reference trajectory $\theta_{\text {nom }}$ with parameters $a$ (amplitude scale), $b$ (amplitude offset) and $T$ (time-stretch) as:

$$
\theta_{\text {adapt }}=a \times \theta_{\text {nom }}(t / T)+d .
$$


If $a=1, d=0, T=1, \theta_{\text {adapt }}$ is the normal gait. The nominal reference trajectory $\theta_{\text {nom }}$ could be online updated to the new satisfactory reference trajectory $\theta_{\text {adapt }}$ adaptively. The modified trajectory $\theta_{\text {new }}$ responding to the patient intention can be described in $\theta_{n o m}$ and $\Delta \theta$ by the following equation:

$$
\theta_{\text {new }}=\theta_{\text {nom }}+\sigma \times \Delta \theta
$$

where $\sigma$ in (23) serves as a scaling parameter to alter the degree of fitness. The trajectory change $\Delta \theta$ is obtained through an inversion of an impedance function as:

$$
\Delta \theta=\frac{1}{K_{P}+K_{D} s} \cdot \tau_{a c t}
$$

where $\tau_{a c t}$ is the extracted active interaction torques between the robot and patient. The adaptation of the trajectory parameters is performed via minimization of the following functions:

$$
M=\min \int_{0}^{T}\left\|\theta_{\text {new }}-\theta_{\text {adapt }}\right\|_{2}^{2} d t,
$$

where $M$ is the optimal function index.

Since the 3 joints (hip joint, knee joint and ankle joint) have the same movement cycle, final time-stretch $(T)$ is set as the average value of the 3 joint $T$ parameters. $a, d$ and $T$ are updated once a cycle to adapt to new trajectory. In the following, we assume that the interaction torques $\tau_{\text {act }}$ results from a virtual spring-like coupling between the desired and the actual position of the robot brace.

\section{Adams Simulation Results}

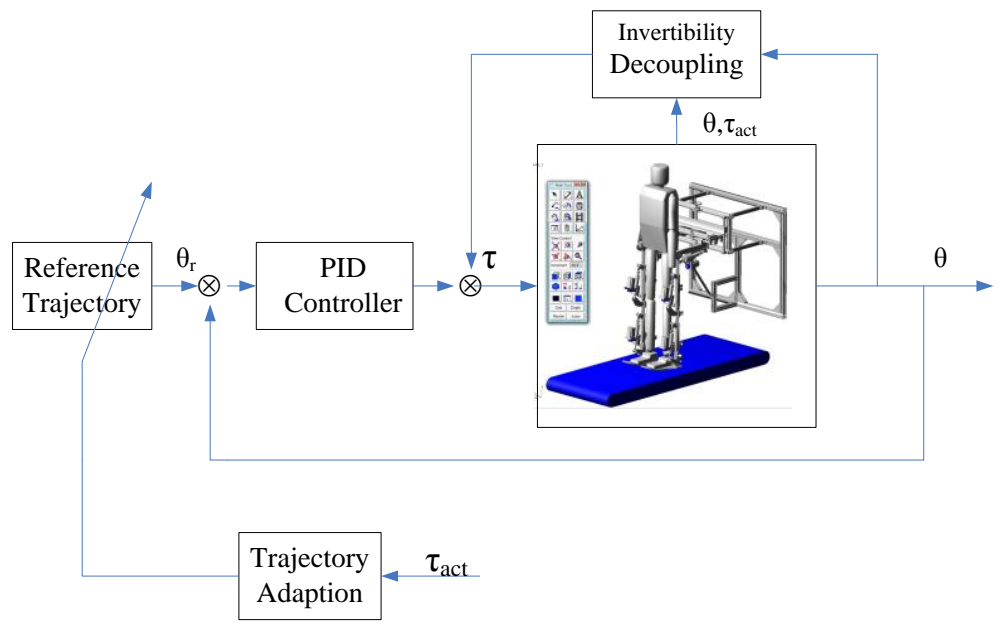

Figure 6. Control block diagram of LERR in Adams and MATLAB/Simulink

The lower extremity rehabilitation robot model is developed in Adams with MATLAB/Simulink environment (in Figure 6), where the human-robot interaction model 
based on the physical parameters of LERR is used to verify the control algorithm. In the following, the performance of the proposed control strategies is presented.

Firstly, the dynamic model of the robot system (1) is assumed to be exactly known, a standard PID controller is developed for each sub-system to track the gait reference trajectory. With the adaptive control strategy, the patient walks in accordance with the standard gait first, and then a patient-robot interaction force is applied at the $30^{\text {th }}$ second. The resulting trajectory of the knee joint is shown in Figure 7. The stable trajectory could be obtained after the $60^{\text {th }}$ second. The trends of the new joint trajectories are the same to the desired ones. The corresponding knee joint torque is shown in Figure 8. The adjusted parameters of new trajectories are shown in Fig. 9. The results show that adaptive control strategy can achieve the modification of the parameters online successfully. Due to the perfect decoupling results of the three joints, only the results of the knee joint are presented here. However, if the dynamic model of the robot system (1) is assumed to be not perfect accurate, for example, assume the weight of the robot and the patient increase $10 \%$ and $30 \%$, respectively, then it is obvious that the resulting system using the controller (5) will not be fully decoupled. In this case, the above specifically designed PID controller with respect to each sub-system could not achieve a good performance for the overall system, and in some situations, the output of tracking trajectories could even diverge as shown in Fig. 10. Considering the uncertainty parameters in the model, and assume $\|\Delta\|_{\infty} \leq 1.13$, then the uncertain weight function $w_{\Delta}$ is selected as

$$
w_{\Delta}=\frac{7.977 \times 10^{-9} s^{2}+0.0001197 s+0.7977}{s^{2}-0.08074 s+0.004723} .
$$

The low pass filter $w(s)$ is selected as

$$
w(s)=\frac{1}{0.1764 s^{2}+0.84 s+1} .
$$

Using these parameters, the proposed iterative algorithm provides the following PID controller

$$
k_{p}=2.97, \quad k_{i}=1.27, \quad k_{d}=0.093
$$

Based on the above designed robust PID controller, the control performance is shown in Figures 11-12. The result of the trajectories of three joints with the adaptive control strategy using robust PID controller is shown in Figure 11. The corresponding adjusted parameters of knee angle trajectories are shown in Figure 12. The obtained results show that the adaptive control strategy with the robust PID controller has achieved successfully a good tracking performance despite the model uncertainty and, consequently, the substantial adaptation that led to reduction of interaction torques between the robot and the patient. 


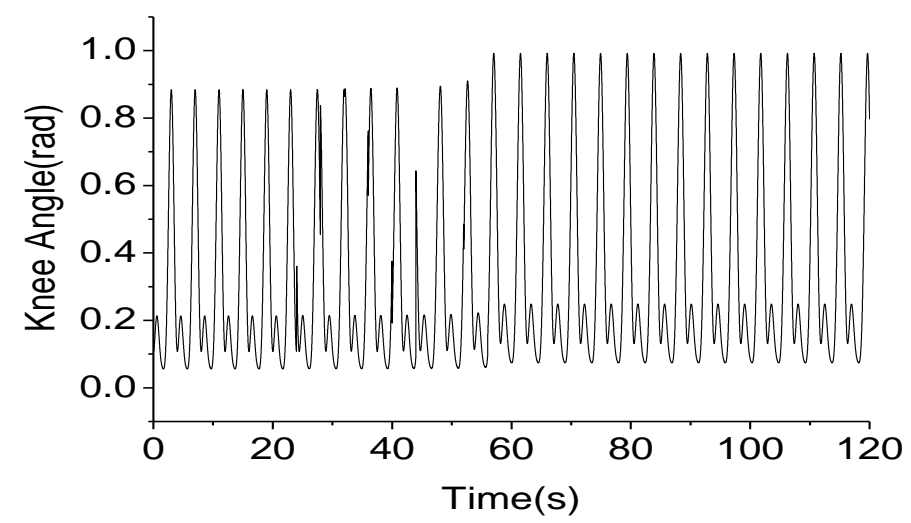

Figure 7. The result of the adaption in the knee joint angle with the adaptive control strategy using standard PID controller without model uncertainty

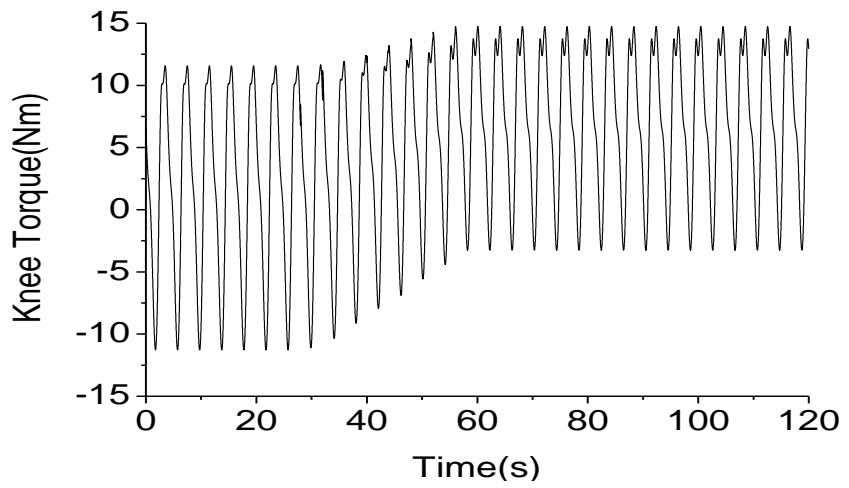

Figure 8. The result of the adaption in the knee joint torque with the adaptive control strategy using standard PID controller without model uncertainty
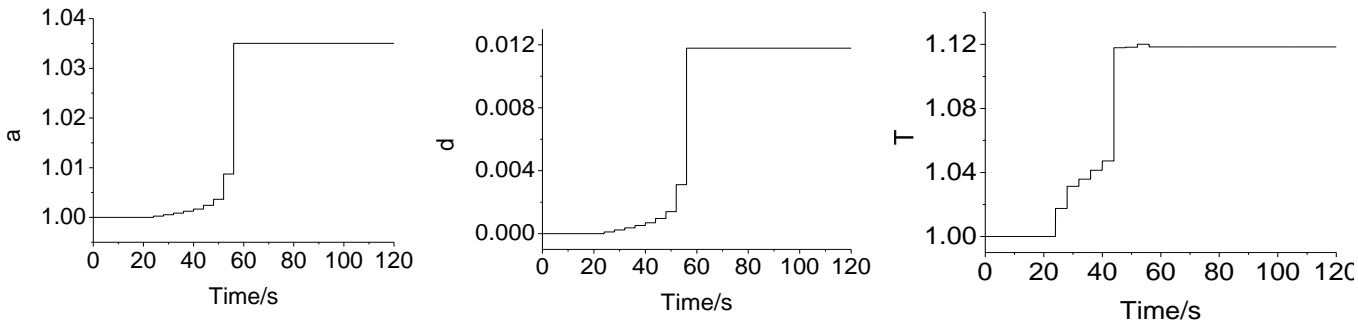

Figure 9. The result of the adaption in the 3 knee joint parameters with the adaptive control strategy using standard PID controller without model uncertainty 


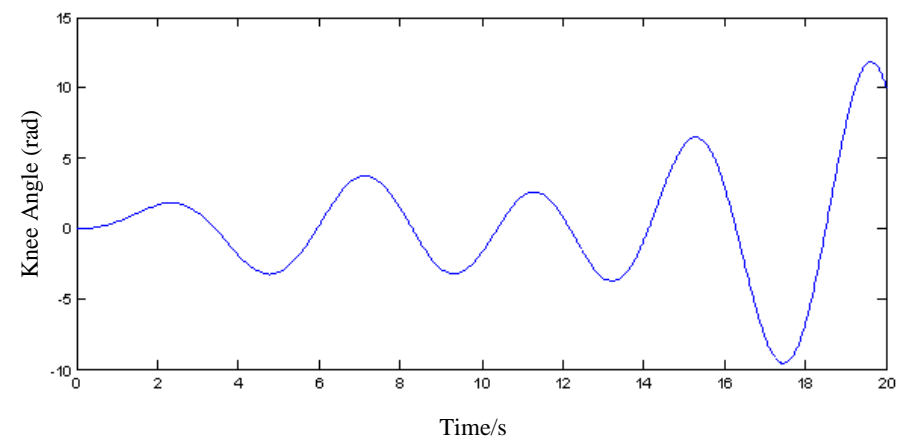

Figure 10. The result of the trajectory of knee joint angle using the standard PID controller with model uncertainty
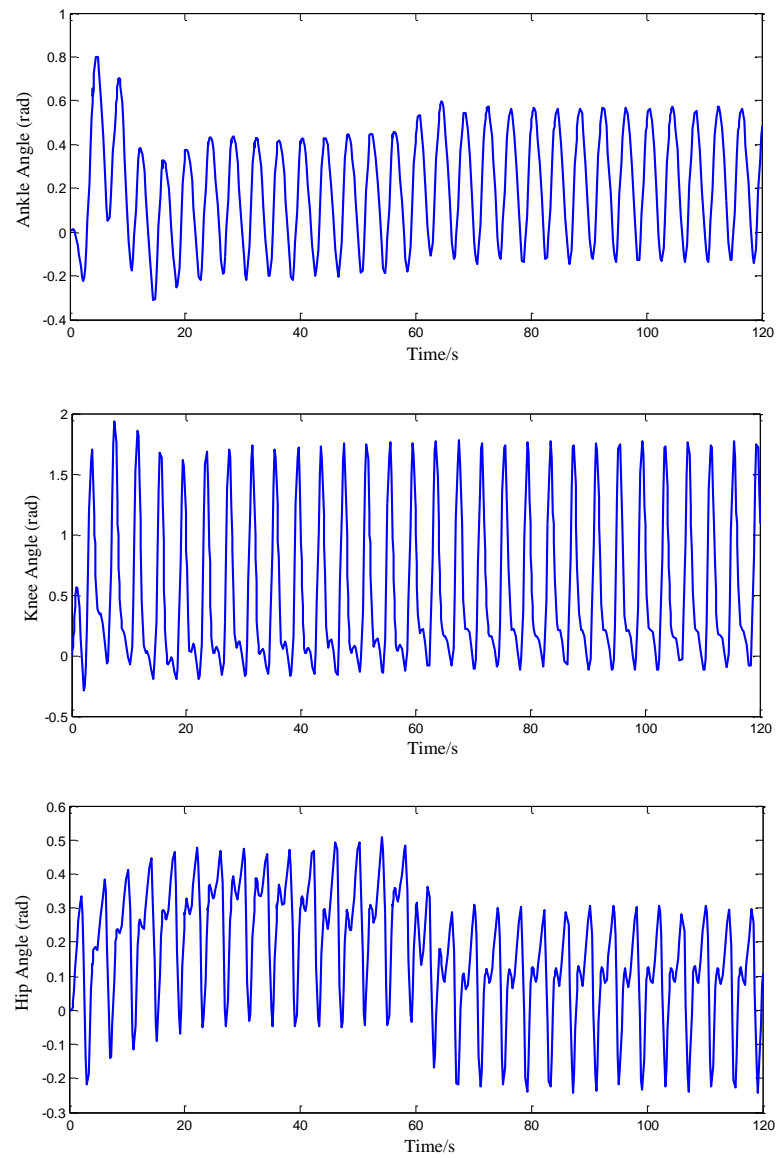

Figure 11. The result of the trajectories of three joints with the adaptive control strategy using robust PID controller with model uncertainty 

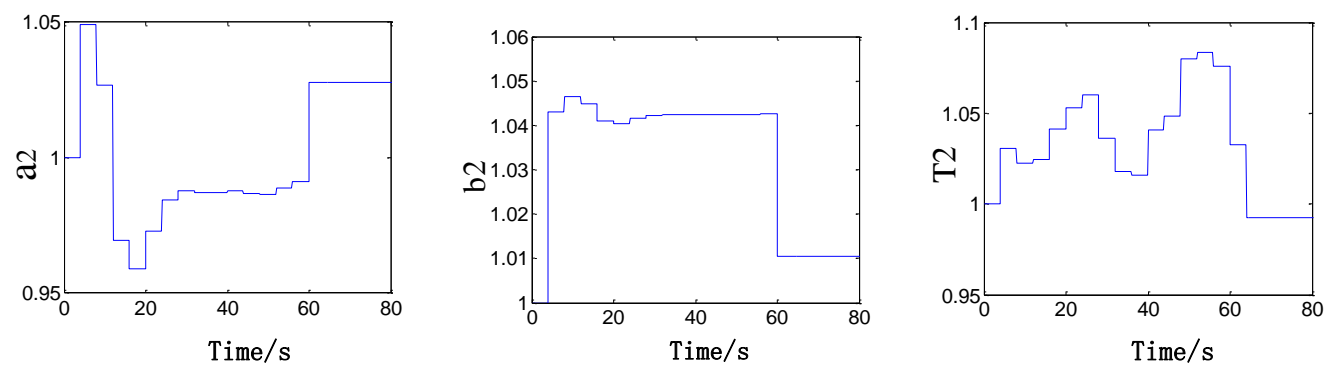

Figure 12. The result of the adaption in the 3 knee joint parameters with the adaptive control strategy using robust PID controller with model uncertainty

\section{Conclusion}

A robust control approach is proposed in this paper to deal with the patient-cooperative strategies for the Lower Extremity Rehabilitation Robot. The invertibility decoupling theory is first applied to convert the nonlinear dynamic model of LERR into some independent two order integral sub-systems, based on which a robust PID controller is designed under the consideration that there exists model uncertainty in the robot system. In order to take into account the patient's intention and voluntary efforts, adaptive control is further developed to adapt the patient cooperatives by online modifying the gait pattern. The satisfactory performance of the control algorithms was verified in Adams-MATLAB/Simulink environment. The results show that the 3 gait trajectories can be effectively controlled and modified online to adapt the patient's active movement intention even with model uncertainty.

\section{Acknowledgments}

This work was supported by the National Natural Science Foundation of China (51075254, 51375289, 5097165) and the Innovation Program of Shanghai Municipal Education Commission (14ZZ092).

\section{References}

[1] V. Dietz, "Neuronal Plasticity after a Human Spinal Cord Injury: Positive and Negative Effects", Experimental Neurology, vol. 235, no. 1, (2012), pp. 110-115.

[2] H. I. Krebs and B.T. Volpe, "Rehabilitation Robotics", Handbook of Clinical Neurology, edited by PJ Vinken and GW Bruyn, vol. 110, (2013), pp. 283-294.

[3] R. Riener, L. Lünenburger, I.C. Maier, G. Colombo, and V. Dietz, "Locomotor Training in Subjects with Sensor-motor Deficits: an Overview of the Robotic Gait Orthosis Lokomat", Journal of Healthcare Engineering, vol. 1, no. 2, (2010), pp. 197-216.

[4] R. Riener, L. Lünenburger, S. Jezernik, M. Anderschitz, G. Colombo and V. Dietz, "Patient-cooperative Strategies for Robot-aided Treadmill Training: First Experimental Results", IEEE Transactions on Neural Systems and Rehabilitation Engineering, vol. 13, no. 3, (2005), pp. 380-394.

[5] M. Pietrusinski, I. Cajigas G. Severini; P. Bonato and C. Mavroidis, "Robotic Gait Rehabilitation Trainer", IEEE/ASME Transactions on Mechatronics, vol. PP, no. 99, (2013), pp. 1-10.

[6] Y. H. Yin, Y. J. Fan and L.D. Xu, "EMG and EPP-integrated Human-machine Interface Between the Paralyzed and Rehabilitation Exoskeleton", IEEE Transactions on Information Technology in Biomedicine, vol. 16, no. 4, (2012), pp. 542-549. 
[7] B. Koopman, E. H. van Asseldonk and H. van der Kooij, "Selective Control of Gait Subtasks in Robotic Gait Training: Foot Clearance Support in Stroke Survivors with a Powered Exoskeleton", Journal of NeuroEngineering and Rehabilitation, doi:10.1186/17430003-10-3, vol. 10, no. 3, (2013).

[8] L. Marchal-Crespo, "Control Strategies for Robot-assisted Training - Literature Review and Experimental Impressions", Converging Clinical and Engineering Research on Neurorehabilitation, Springer Berlin Heidelberg, (2013), pp. 115-120.

[9] Z. Feng, J. Qian, Y. Zhang and L. Shen, "Dynamics Modeling and Motion Control for Lower Limb Exoskeleton Orthosis", Chinese High Technology Letters, doi:10.3772/j.issn.1002-0470.2009.03.010, vol. 19, no. 3, (2009).

[10]S. N. Sign, "Decoupling of Invertible Nonlinear Systems with State Feedback and Precompensation", IEEE Transactions on Automat Control, vol. 25, no. 6, (1980), pp. $1237-1239$

[11]S. N. Sigh, "A Modified Algorithm for Invertibility in Nonlinear systems", IEEE Transactions on Automat Control, vol. 26, no. 2, (1981), pp. 595-598.

[12]K. Zhou, J. Doyle and K. Golver, "Robust and Optimal Control, Prentice Hall", Upper Saddle River, (1995).

[13]A. Iqbal, Z. Wu and F. Ben Amara, "A Decentralized Robust PID Controller Design for the Shape Control of a Magnetic Fluid Deformable Mirror", International Journal of Optomechatronics, vol. 4, (2010), pp. 246-268.

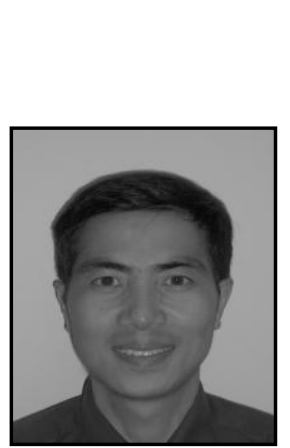

\section{Authors}

\section{Zhizheng Wu}

Zhizheng Wu received the B.S. degree and M.S. degree in Electrical and Electronic Engineering from Hunan University, China, in 1993 and 1995, the Ph.D. degree in Electronic and Information Engineering from Shanghai Jiaotong University, China, in 1998, and the Ph.D. degree in Mechanical Engineering from the University of Toronto, Canada, in 2008. From 1999 to 2004, he was with Innomedia, Inc. Shanghai, Hongkou Information Committee of Shanghai Government and Omron Dualtec Automotive Electronics, Inc. Toronto, respectively. From 2004 to 2010, he was a Research Associate with the Department of Mechanical and Industrial Engineering at the University of Toronto. He is now an associate professor in the Department of Precision Mechanical Engineering at Shanghai University. His research interests include adaptive control; robust control; optomechatronic systems; Robotics.

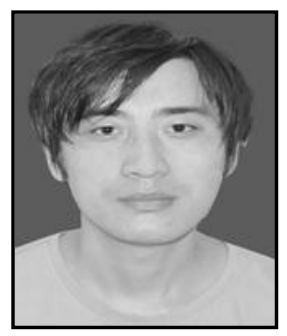

\section{Feng Li}

Feng Li is currently a $\mathrm{PhD}$ candidate in Mechanical Engineering at Shanghai University, China. His research interests include man-machine system and control of medical rehabilitation robot. 


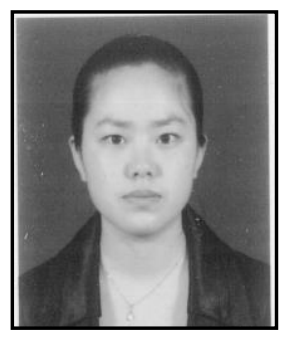

\section{Wenjun Mi}

Wenjun $\mathrm{Mi}$ is a master student in Mechatronic Engineering at Shanghai University, China. Her main research interests include the control of medical rehabilitation robot and mechatronic system design.

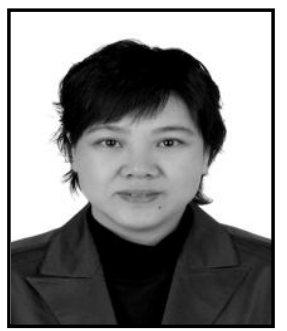

\section{Jingtao Lei}

Jingtao Lei received her PhD degree in Mechanical Engineering from Beihang University, China, in 2007. She is currently an associate professor at School of Mechatronic Engineering and Automation, Shanghai University, China. Her research interests include walking robots and robot modular technology. 